\title{
Evaluation of Actical equations and thresholds to predict physical activity intensity in young children
}

Xanne Janssen $^{1 *}$, Dylan P Cliff ${ }^{1}$, John J. Reilly ${ }^{2}$, Trina Hinkley ${ }^{3}$, Rachel A. Jones ${ }^{1}$, Marijka Batterham $^{4}$, Ulf Ekelund ${ }^{5,6}$, Søren Brage ${ }^{6}$ and Anthony D. Okely ${ }^{1}$.

1. Interdisciplinary Educational Research Institute, University of Wollongong, Wollongong, New South Wales, Australia, 2522.

2. School of Psychological Sciences and Health, University of Strathclyde, Glasgow, Scotland, G1 1XQ.

3. Centre for Physical Activity and Nutrition Research (C-PAN), Deakin University, Melbourne, Victoria, Australia, 3125

4. Centre for Statistical and Survey Methodology, University of Wollongong, Wollongong, New South Wales, Australia, 2522.

5. Department of Sport Medicine, Norwegian School of Sport Sciences, Ullevål Stadion, Oslo, Norway, 0806.

6. MRC Epidemiology Unit, Institute of Metabolic Science, Addenbrooke's Hospital, Cambridge, United Kingdom, CB2 0QQ.

*Author for correspondence and guarantor:

Xanne Janssen

Faculty of Education, University of Wollongong

Northfields Avenue, Wollongong, NSW, 2522 Australia

Email: xj512@uowmail.edu.au; Phone: +61 2 42392398; Fax: +61 242213892

Running title: Validity of Actical energy expenditure equations and cut-points Keywords: accelerometry; room calorimeter; preschoolers 


\section{Abstract}

This study examined the validity of current Actical activity energy expenditure equations and intensity cut-points in preschoolers using activity energy expenditure and direct observation as criterion measures. Forty $4-6$ year-olds $(5.3 \pm 1.0$ years $)$ completed a $\sim 150$ min room calorimeter protocol involving age-appropriate sedentary behaviours, light intensity physical activities and moderate-to-vigorous intensity physical activities. Activity energy expenditure and/or physical activity intensity were calculated using Actical equations and cut-points by Adolph, Evenson, Pfeiffer and Puyau. Predictive validity was examined using paired sample t-tests. Classification accuracy was evaluated using weighted kappas, sensitivity, specificity, and area under the receiver operating characteristic curve. The Pfeiffer,equation significantly overestimated activity energy expenditure during sedentary behaviour and underestimated activity energy expenditure during light intensity physical activity $(\mathrm{P}<0.0125$ for both). There was no significant difference between measured and predicted activity energy expenditure during moderateto-vigorous intensity physical activity. The Adolph cut-point showed significantly higher accuracy for classifying sedentary behaviour, light intensity physical activity and moderate-to-vigorous intensity physical activity than all others. The available Actical equation does not provide accurate estimates of activity energy expenditure across all intensities in preschoolers. However, the Pfeiffer equation performed reasonably well for moderate-to-vigorous intensity physical activity. Using cut-points of $\leq 6$ counts $\cdot 15 \mathrm{~s}^{-1}, 7-286$ counts $\cdot 15 \mathrm{~s}^{-1}$ and $\geq 287$ counts $\cdot 15 \mathrm{~s}^{-1}$ when classifying sedentary behaviour, light intensity physical activity and moderate-to-vigorous intensity physical activity, respectively, is recommended.

\section{Introduction}


Reports on the prevalence of physical activity and sedentary behaviour in young children using objective measures have been inconsistent (Cardon \& De Bourdeaudhuij, 2008; Hinkley, 2012; Vale, Silva, Santos, Soares-Miranda, \& Mota, 2010; Van Cauwenberghe, Jones, Hinkley, Crawford, \& Okely, 2012). This might be due, in part, to variability in the methods used to measure physical activity and sedentary behaviour (Beets, Bornstein, Dowda, \& Pate, 2011; Oliver, Schofield, \& Kolt, 2007). Accelerometry is the method of choice for assessing physical activity and sedentary behaviour objectively in free-living conditions among young children (Trost, 2007) and the Actical accelerometer is one of the most commonly used accelerometers in such studies (Carter, Taylor, Williams, \& Taylor, 2011; Colley et al., 2011; Dolinsky, Brouwer, Østbye, Evenson, \& Siega-Riz, 2011). However, one issue faced by Actical users is that scarce evidence is available to assist in deciding how to calculate activity energy expenditure or define physical activity intensity from the multiple available equations or cut-point definitions (Adolph et al., 2012; Evenson, Catellier, Gill, Ondrak, \& McMurray, 2008; Pate, Almeida, McIver, Pfeiffer, \& Dowda, 2006; Pfeiffer, McIver, Dowda, Almeida, \& Pate, 2006; Puyau, Adolph, Vohra, Zakeri, \& Butte, 2004).

Several studies have developed activity energy expenditure equations and cut-points specific to the Actical accelerometer, based on accelerometer counts, to estimate activity energy expenditure or classify physical activity intensity (Adolph, et al., 2012; Evenson, et al., 2008; Pfeiffer, et al., 2006). However, key methodological differences exist in the studies from which those equations and cut-points were developed (Table 1). For instance, one study included only sitting, walking and running (Pfeiffer, et al., 2006) where as others have included free-living activities which may be more representative of children's behaviours (e.g. playing with toys, arts and crafts, stair walking) (Adolph, et al., 2012; 
Evenson, et al., 2008). These variations during calibration of the Actical may explain why different equations and cut-points for estimating activity energy expenditure and classifying physical activity intensity have emerged (Adolph, et al., 2012; Evenson, et al., 2008; Pfeiffer, et al., 2006).

The use of different equations or cut-points may subsequently result in differences in children's predicted activity energy expenditure or estimated time in sedentary behaviour and physical activity intensities, making it hard to compare findings between studies (Cliff \& Okely, 2007; Reilly et al., 2008; Van Cauwenberghe, Trost, De Bourdeaudhuij, \& Cardon, 2011). These inconsistencies limit the ability of policy makers, public health officials and the general public to determine young children's compliance with physical activity guidelines (Beets, et al., 2011). The activity energy expenditure equations and cutpoints developed for the Actical monitor need to be cross-validated against appropriate criterion measures to overcome this methodological limitation. However, no such studies demonstrating the most accurate Actical equations and cut-points among 4-6 year-old children are evident in the extant literature. Therefore, the aims of this study were to: 1) examine the predictive validity of Actical activity energy expenditure equations; and 2) compare the classification accuracy of existing Actical cut-points for classifying physical activity and sedentary behaviour intensity, in 4-6 year-old children using activity energy expenditure measured by room calorimetry and physical activity intensity classified by direct observation as criterion measures.

\section{Methods}

\subsection{Study participants}


Forty participants between the ages of 4-6 years were recruited from childcare centres (preschools, long-day and family-day care) in the Illawarra region of New South Wales, Australia. Children with a disease known to influence their energy balance, physical disabilities and/or claustrophobia were excluded from this study. The study was approved by the University of Wollongong/ South Eastern Sydney and Illawarra Area Health Service Human Research Ethics Committee. Parents provided informed written consent, and children provided their verbal assent to participate in the study.

\subsection{Protocol}

Participants visited the laboratory twice. During the first visit, participants and their parents were familiarized with the room calorimeter and the activity protocol. Visit two occurred within a week after the first visit. Parents were asked to give their child a light standardized breakfast 1.5 hour before entering the room calorimeter. During the second visit participants followed a 150-minute activity protocol within the room calorimeter. The protocol involved child-appropriate sedentary behaviours, light intensity physical activities and moderate-to-vigorous intensity physical activities . All children were guided through the protocol by a research assistant and performed each activity, for a pre-determined duration, in identical order (online supplement 1). The protocol followed current best practice recommendations for validation studies in that it included a variety of activities that are developmentally appropriate for the age group and that range from sedentary behaviours to moderate-to-vigorous intensity physical activities (Bassett, Rowlands, \& Trost, 2012).

\subsection{Room Calorimeter}


Oxygen consumption $\left(\mathrm{VO}_{2}\right)$ and carbon dioxide production $\left(\mathrm{VCO}_{2}\right)$ were measured continuously (paramagnetic $\mathrm{O}_{2}$ and infrared $\mathrm{CO}_{2}$ analysers, Sable System Inc., Las Vegas USA) and corrected to standard temperature, pressure and humidity in a room calorimeter $(3 \mathrm{~m} \times 2.1 \mathrm{~m} \times 2.1 \mathrm{~m})$. Technical procedures are described in more detail elsewhere(Janssen et al., 2013). Chamber air was sampled every two minutes and rates of $\mathrm{O}_{2}$ consumption and $\mathrm{CO}_{2}$ production were then averaged over 10 -minute blocks to produce stable measures of energy expenditure (Schoffelen, Westerterp, Saris, \& Ten Hoor, 1997).

\subsection{Direct observation of PA intensity}

Each child was videotaped during the protocol and activity start and end times, breaks and transitions were recorded. PA intensity was classified using the Children's Activity Rating Scale (Puhl, 1990). The Children's Activity Rating Scale is based on a 1 to 5 coding scheme and is a reliable and valid tool to assess physical activity levels in young children (DuRant, Baranowski, Puhl, \& Rhodes, 1993). It has been used in several accelerometer validation studies in young children (De Bock et al., 2010; Van Cauwenberghe, et al., 2011). Video footage was coded with the help of Vitessa 0.1 (Version 0.1, University of Leuven, Belgium) which generated a time stamp every time a change in intensity was coded by the observer (Van Puyenbroeck, Maes, \& Laeremans, 2005). Data were coded by one observer who undertook two days of specific training using the Children's Activity Rating Scale. During training, data from pilot trials were used. After coding, a weighted average Children's Activity Rating Scale score was calculated over 15-second or 60second epochs corresponding to the Actical measurement epochs. Averaged epochs were then classified into intensity categories using the Children's Activity Rating Scale criteria: sedentary behaviour < level 2.0; light intensity physical activity $\geq$ level 2.0 and $\leq 3.0$; moderate-to-vigorous intensity physical activity > level 3.0 (Puhl, 1990). 


\subsection{Accelerometry}

Before entering the room calorimeter children were fitted with an Actical (Philips Respironics, Bend, OR) accelerometer. The Actical was worn on the right mid-axillary line of the hip. Accelerometers were initialized to collect data in 15-second epochs.

\subsection{Data reduction}

The room calorimeter, Actical and camcorder were time synchronized on the morning of every measurement day. Energy expenditure for every 10-minute block was calculated using the Weir equation (Weir, 1949). Individualized multiples of resting energy expenditure ( metabolic equivalents) were calculated by dividing measured energy expenditure for each child by their individually estimated basal metabolic rate using the Schofield equation for children aged 4-10 years (Schofield, 1985). The 10-minute blocks of energy expenditure were classified, based on their metabolic equivalent values, into physical activity intensities as follows; sedentary behaviour $\leq 1.5$ times predicted basal metabolic rate, light intensity physical activity $>1.5$ to $<3.0$ times predicted basal metabolic rate and moderate-to-vigorous intensity physical activity $\geq 3.0$ times predicted basal metabolic rate. As there is still inconsistency between the use of 3 or 4 times resting energy expenditure as moderate-to-vigorous intensity physical activity threshold in children (Cain et al. 2013), and most cut points in this study have been developed using a threshold of 3 times resting energy expenditure, this was used. Activity energy expenditure was determined by deducting basal metabolic rate, calculated using the Schofield equation, from measured energy expenditure. Actical output and direct observation data were used as 15-second epochs or converted to 60-second epochs depending on the equation/cut-point as determined from the original study. 
Prediction of AEE. Actical counts were converted to $\mathrm{VO}_{2}$ and activity energy expenditure using the Pfeiffer (Pfeiffer, et al., 2006) (Table 1) and averaged over 10-minute blocks. $\mathrm{VO}_{2}$ values are converted to activity energy expenditure to facilitate comparison. To our knowledge, the equations developed in preschool aged children were limited to the Pfeiffer equation. The Adolph and Evenson cut-points do not have an associated activity energy expenditure equation and therefore these were not included in assessing the validity of activity energy expenditure predictions (Adolph, et al., 2012; Evenson, et al., 2008). Participants' activity energy expenditure data were averaged per intensity and over the duration of the protocol. Predicted activity energy expenditure values were then compared to measured activity energy expenditure values by the room calorimeter.

Prediction of PA intensity and SB. Actical data were classified as sedentary behaviour, light intensity physical activity and moderate-to-vigorous intensity physical activity using the Pfeiffer, Adolph and Evenson Actical cut-points. Pfeiffer et al. (2006) only developed moderate-to-vigorous intensity physical activity cut-point and therefore no sedentary behaviour/light intensity physical activity cut-point was available. The Children's Activity Rating Scale and Actical data were used as 15 -second epochs or 60 -second epochs. Several studies indicate that epochs shorter than 60 seconds should be used in young children to avoid underestimating their moderate-to-vigorous intensity physical activity, due to their sporadic and intermittent physical activity behaviour (McClain, Abraham, Brusseau Jr, \& Tudor-Locke, 2008; Reilly, et al., 2008). As such, in case of a cut-point being developed as 60 -second epoch, the cut-point was reanalysed as 15 -second epoch by dividing the original cut-point by 4 . 
Direct observation systems such as the Children's Activity Rating Scale rely on subjective classification and use general category descriptions to assign levels to activities based on the apparent intensity of the activity. However, a large variability in energy expenditure has been shown in young children during these activities (Pate, et al., 2006; Trost, Loprinzi, Moore, \& Pfeiffer, 2011). Therefore, in this study we developed a new criterion measure, combining the Children's Activity Rating Scale with measured activity energy expenditure from the room calorimeter, to classify the intensity of criterion epochs. Ten minute average energy expenditure values were divided by predicted basal metabolic rate to define intensity levels. Each of the forty 15 -second epochs or ten 60 -second epochs within the 10-minute period immediately prior to the measured average energy expenditure value were classified as sedentary behaviour, light intensity physical activity, or moderateto-vigorous intensity physical activity according to the calculated metabolic equivalent. Direct observation data were averaged over the same 15 or 60 second periods' cut-point to define intensity levels for each epoch. Thereafter, criterion epochs were excluded if physical activity intensity defined using energy expenditure measured by the room calorimeter did not agree with the intensity levels derived via direct observation for each epoch. In addition, to ensure that the time lag in the calorimeter readings of expired air would not lead to mismatching criterion data with accelerometer data, epochs within the last minute of a 10-minute energy expenditure data block were excluded. Likewise, criterion epochs that occurred during transitions between activities were excluded as the aim was to examine classification accuracy using specific activities appropriate for young children. Actical data were classified as sedentary behaviour, light intensity physical activity, or moderate-to-vigorous intensity physical activity as previously described. Classified Actical data were then compared with room calorimeter and direct observation data. 


\section{7 $\underline{\text { Statistical analysis }}$}

To examine the predictive validity of the Actical equations, differences between measured activity energy expenditure and predicted activity energy expenditure were compared using dependent $t$-tests with a Bonferroni adjustment for multiple comparisons $(\mathrm{P}<0.0125)$. In addition, root means square errors were calculated. To evaluate classification accuracy of

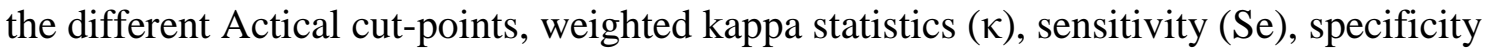
(Sp), and area under the receiver operating characteristic curve (ROC-AUC) were calculated. ROC-AUC values were defined as excellent (0.9-1.0), good (0.8-0.9), fair (0.7$0.8)$, or poor $(<0.7)($ Metz, 1978), whereas $\kappa$ was defined as having slight $(0-0.20)$, fair (0.21-0.40), moderate (0.41-0.60), substantial (0.61-0.80), and almost perfect (0.81-1.00) agreement (Landis \& Koch, 1977). All statistical analyses were performed using STATA Version 12 (StataCorp, College Station, Texas, USA).

\section{Results}

A sample of 40 children completed both visits; two had missing data due to calorimeter malfunction. For the remaining 38 children, 33 (86.8\%), $36(94.7 \%)$, and $34(89.5 \%)$ each had at least one 10-minute block of sedentary behaviour, light intensity physical activity, and moderate-to-vigorous intensity physical activity, respectively, according to measured energy expenditure values. For sedentary behaviour, light intensity physical activity, and moderate-to-vigorous intensity physical activity measured-average (range) metabolic equivalent values were 1.1 (1-1.5), 1.9 (1.5-2.5), and 3.7 (3.0-5.5), respectively. Descriptive characteristics and median energy expenditure values per intensity are presented in Table 2 and 3. 
Prediction of AEE. Observed and predicted activity energy expenditure values for the Pfeiffer equations are shown in Table 4. The Pfeiffer equation significantly overestimated activity energy expenditure during sedentary behaviour and significantly underestimated activity energy expenditure during light intensity physical activity $(\mathrm{P}<0.0125$ for all). However, Pfeiffer did not show a significant difference between measured and predicted activity energy expenditure during moderate-to-vigorous intensity physical activity or total activity energy expenditure ( $\mathrm{P}=0.76$ and 0.80 , respectively).

Prediction of PA and SB intensity using direct observation. When using direct observation as the criterion method, $84.8 \%$ (18953 epochs) of the available data was included in the analyses. Data was excluded due to child being off screen (3397 epochs). For sedentary behaviour this was $96.3 \%$ (6881 epochs), for light intensity physical activity this was $96.4 \%$ (7325 epochs) and for moderate-to-vigorous physical activity this was $62.5 \%$ (4747 epochs).

Overall, Adolph $(\kappa=0.64,95 \% \mathrm{CI}=0.64-0.65)$ exhibited substantial agreement. Adolph $15 \mathrm{~s}$ $(\kappa=0.58,95 \% \mathrm{CI}=0.57-0.58)$, and Evenson $(\kappa=0.52,95 \% \mathrm{CI}=0.52-0.53)$, exhibited moderate agreement. For each cut-point, sensitivity, specificity and ROC-AUC were analysed for sedentary behaviour, light intensity physical activity, and moderate-tovigorous intensity physical activity. Results are reported in Table 5. For sedentary behaviour, classification accuracy was significantly higher for Adolph compared to all others $(\mathrm{P}<0.05)$. Classification accuracy was good for (ROC-AUC $=0.82$, respectively), whereas Evenson exhibited fair classification accuracy (ROC-AUC=0.79). When comparing only 15 second epochs the Adolph cut-point showed good classification accuracy $(\mathrm{ROC}-\mathrm{AUC}=0.80)$, performing significantly better than Evenson $(\mathrm{P}<0.05)$. In 
addition, inverse results were found for light-to-moderate-to-vigorous intensity physical activity.

For light physical activity, the Adolph cut-point exhibited fair classification accuracy (ROC-AUC=0.73), whereas Evenson showed poor classification accuracy (ROCAUC $=0.56-0.66)$. Both cut-points had good specificity (79.0\%-86.8\%). However, Adolph showed a significantly higher sensitivity $(66.2 \%)$, which resulted in significantly higher classification accuracy compared to Evenson $(\mathrm{P}<0.05)$. The results of Adolph ${ }_{15 \mathrm{~s}}$ show a decrease in classification accuracy. However, the Adolph ${ }_{15 s}$ performs significantly better than Evenson.

For moderate-to-vigorous intensity physical activity, the Adolph cut-point exhibited good classification accuracy (ROC-AUC=0.85), whereas Evenson and Pfeiffer cut-points exhibited fair classification accuracy (ROC-AUC $=0.75$ and 0.70 , respectively). Classification accuracy was significantly higher for Adolph compared to all others $(\mathrm{P}<0.05)$. When comparing only 15 second epoch cut-points only the Adolph ${ }_{15 s}$ exhibited good classification accuracy (ROC-AUC $=0.82$ ) and performed significantly better than all others $(\mathrm{P}<0.05)$.

Prediction of $P A$ and $S B$ intensity using direct observation and EE. When using direct observation in conjunction with measured energy expenditure by the calorimeter as the criterion measure, $58.4 \%$ (13052 epochs) of the available data was included in the analyses. For sedentary behaviour 70.5\% (5036 epochs) was included, for light intensity physical activity this was $81.8 \%$ (6214 epochs), and for moderate-to-vigorous intensity physical activity this was $23.7 \%$ (1802 epochs). Data exclusion was due to lack of 
agreement between calorimeter and direct observation data (5901epochs) and the child being off screen (3397 epochs). Overall $\kappa$ values were slightly increased compered to using direct observation only. Adolph $(\kappa=0.72,95 \% \mathrm{CI}=0.70-0.74), \operatorname{Adolph}_{15 \mathrm{~s}}(\kappa=0.65,95 \%$ $\mathrm{CI}=0.64-0.65)$ and Evenson $(\kappa=0.6195 \% \mathrm{CI}=0.60-0.62)$ exhibited substantial agreement. ROC-AUC values when using direct observation combined with energy expenditure were slightly higher but nevertheless similar to using direct observation only (Table 6). Adolph was significantly better than all others $(\mathrm{P}<0.05)$ when classifying light intensity physical activity and moderate-to-vigorous intensity physical activity. For sedentary behaviour, classification accuracy was good for the Adolph and Evenson (ROC-AUC=0.82-0.85) with Adolph performing significantly better than Evenson. However, when comparing Evenson and Adolph 15 s this difference disappeared.

\section{Discussion}

This is the first study to report on the predictive validity of Actical activity energy expenditure equations and cut-points in 4-6 year-olds. The Pfeiffer equation significantly overestimated activity energy expenditure during sedentary behaviour and underestimated light intensity physical activity, but demonstrated greater accuracy for predicting activity energy expenditure during moderate-to-vigorous intensity physical activity and total activity energy expenditure.

To our knowledge the Pfeiffer equation has only been cross-validated in preschool aged children once (Pfeiffer, et al., 2006). Pfeiffer, et al. cross-validated the equation for estimating energy expenditure during moderate-to-vigorous intensity physical activity and found an underestimation of activity energy expenditure during moderate-to-vigorous intensity physical activity. These results are similar to the findings in this study. In 
addition, the current study found significant differences between predicted and measured activity energy expenditure during sedentary behaviour and light intensity physical activity for Pfeiffer. This is not surprising, as the equation and associated cut-points were developed to estimate and classify moderate-to-vigorous intensity physical activity (Pfeiffer, et al., 2006).

Previously, it has been shown that single-regression equations do not accurately estimate energy expenditure across all physical activity intensities (Bassett et al, 2012). It has been suggested that alternative models such as those involving two regression equations might perform better compared to single regression equations (Bassett et al, 2012). Therefore, we conducted a sub-analysis examining the validity of the Actical energy expenditure prediction model developed by Heil et al. (2003) involving two regression equations; one for the lower intensities of physical activity and one for moderate-to-vigorous physical activity. Despite the fact that this equation was developed in adolescents, the results showed that the accuracy for estimating activity energy expenditure during light intensity physical activity and moderate-to-vigorous intensity physical activity increased (see online supplement 2). This confirms that the relationship between accelerometer counts and activity energy expenditure is not linear and perhaps other approaches, such as tworegression models or those based on pattern recognition techniques should be explored further.

The second aim of this study was to investigate the classification accuracy of Actical sedentary behaviour and physical activity cut-points in 4-6 year-old children. For all intensities combined and separate, Adolph and Adolph ${ }_{15 s}$ (when comparing 15-second epoch cut-points only) resulted in significantly greater classification accuracy compared to 
all others. Sensitivity was clearly higher for Adolph and Adolph ${ }_{15 s}$ when classifying moderate-to-vigorous intensity physical activity indicating the Adolph equation underestimates time spent in moderate-to-vigorous intensity physical activity less than all others. As the current physical activity recommendations for young children are operationalised as time spent in light-to-moderate-to-vigorous intensity physical activity (Department of Health, 2011; Department of Health and Ageing, 2009; Tremblay et al., 2012) this is of special interests in this age group. The Adolph ${ }_{15 \text { s }}$ showed good classification accuracy for light-to-moderate-to-vigorous intensity physical activity. Therefore these findings support the use of Adolph Actical cut-points in 4-6 year-old children when examining the prevalence of children meeting physical activity recommendations. As this is the first study to examine the classification accuracy of Actical physical activity and sedentary behaviour cut-points in 4-6 year-old children comparison to other studies is limited. The cut-points included in the current study were all developed in samples including preschool children (Adolph, et al., 2012; Evenson, et al., 2008; Pfeiffer, et al., 2006). Adolph resulted in the best classification accuracy for all physical activity intensities. Both Pfeiffer and Evenson did not perform as well when classifying moderate-to-vigorous intensity physical activity. A previous study examining the accuracy of the Evenson cut point for the ActiGraph, which was developed simultaneously with the Evenson Actical cut point, reported similar results (Janssen et al., 2013). However, Trost et al. reported that the Evenson cut point was the most accurate cut point among 5-15 year-old children and adolescents. This may indicate that age-related cut point definitions for physical activity intensity might be useful (Trost, 2007). Pfeiffer et al. reported an underestimation of $\mathrm{VO}_{2}$ when cross-validating the developed Pfeiffer equation on which the moderate-to-vigorous intensity physical activity cut-point was based (Pfeiffer, et al., 2006). This is in line with the results of the current study, which suggest 
that the Pfeiffer cut-point might be too high to accurately classify moderate-to-vigorous intensity physical activity. In addition, free-living physical activities were not included in Pfeiffer et al.'s (Pfeiffer, et al., 2006) calibration protocol, whereas Adolph et al. (2012) did include free-living activities. Calibration studies should include ambulatory and nonambulatory activities to cover a broad range of free-living activities (Bassett, et al., 2012). Such reasons may explain why the Pfeiffer cut-point did not perform as well as cut-points developed in studies where the activity protocol included free-living activities (Adolph, et al., 2012; Evenson, et al., 2008).

Several studies indicate that epochs shorter than 60 seconds should be used in young children to avoid underestimating their moderate-to-vigorous intensity physical activity, due to their sporadic and intermittent PA behaviour (McClain, et al., 2008; Reilly, et al., 2008). The results from the present study, which showed better classification accuracy when using the 60-second epochs (i.e. Adolph) are therefore somewhat unexpected. The structured protocol used in the present study protocol might have limited opportunity for the sporadic behaviour that characterizes young children's free-living activities. This might have led to our analyses being less prone to epoch-related misclassification. In addition, the number of epochs included for analysis was greater when using 15-second epochs, resulting in more opportunities for possible miss classification. However, reanalysing the 60-second epochs as 15-second epochs led to similar results. Using the Adolph ${ }_{15 \text { s }}$ cut-point still resulted in the best classification accuracy over all intensities. As such, we recommend using 15-second epochs when measuring physical activity intensity in young children.

This study has several strengths and limitations. The room calorimeter is a confined space and the children followed a standardized activity protocol, limiting the ability to represent 
children's free-living, intermittent physical activity patterns and the generalizability of the findings to free-living conditions. However, the protocol included developmentally appropriate, free-living activities and, due to the small size and stature of the children, the limited space may have had less influence on their activity behaviour than might be the case in older children or adults. As it was considered unfeasible to ask children to fast before following a 2.5 hour activity protocol resting energy expenditure measures were not available and therefore predicted basal metabolic rate was used. This might have influenced the defined metabolic equivalents in this study. However, the use of predicted basal metabolic rate is consistent with similar studies in older children and adolescents (Reilly et al., 2006; Trost, et al., 2011; Trost, Way, \& Okely, 2006). Due to the calorimeter sampling frequency and the time lag that exists when measuring energy expenditure in large volumes, it was not possible to measure energy expenditure in time blocks shorter than 10 min (Schoffelen, et al., 1997), making it impossible to have $15 \mathrm{~s}$ epoch specific energy expenditure values. The use of a Douglas bag, ventilated hood or portable system might have provided a smaller sampling window resulting in a slightly increased accuracy. However, due to the age of the children and the nature of the protocol these methods were impractical. By including direct observation as well as measured energy expenditure by the room calorimeter the impact of potential misclassification errors associated with each of the criterion measures was reduced. In addition, using the room calorimeter made it possible for children to perform activities without being limited by wearing a facemask and portable calorimetry device. Wearing a facemask may potentially impact on the performance of a given activity, especially in young children, who may be less able to sustain the weight and discomfort of a portable device compared to older children, and in young children the extra weight of a portable device may increase the energy cost of activity significantly. Finally, it is possible that cut-point methodology might soon be 
replaced by pattern recognition analysis techniques applied to waveform data (Freedson, Bowles, Troiano, \& Haskell, 2012). However, pattern recognition techniques are still in the developmental stages and until such methodologies are more widely available, the accurate classification of SB and PA using cut-points will remain an important issue for researchers, in particular for those using already collected epidemiological data on child exposures to disease outcomes in adulthood. It is important, therefore, to identify which cut-points are most accurate in young children as it is clear that the use of different cut-points results in substantial differences when reporting time spent sedentary and in each of the physical activity intensities (Cliff \& Okely, 2007; Reilly, et al., 2008; Van Cauwenberghe, et al., 2011), and when estimating compliance with physical activity recommendations (Beets, et al., 2011).

\section{Conclusion}

In summary, the Pfeiffer equation did not accurately predict activity energy expenditure for all physical activity intensities. For moderate-to-vigorous intensity physical activity, the equation did perform well and could possibly be used to predict activity energy expenditure during moderate-to-vigorous intensity physical activity in 4-6 year-old children. However, further assessment in a broader range of typical non-ambulatory activities is required for the equation to be used with confidence across a broad range of free-living physical activity. In addition, other approaches (e.g. multiple regression analysis) might improve accuracy further. When assessing the prediction of physical activitiy intensity, Adolph and Adolph 15 s performed best across all intensities. We recommend using the Adolph ${ }_{15 \text { s }}$ cut-points of $\leq 6$ counts $\cdot 15$ seconds $^{-1}$ when classifying sedentary behaviour, $7-286$ counts $\cdot 15$ seconds $^{-1}$ when classifying light intensity physical 
activity and $\geq 287$ counts $\cdot 15$ seconds ${ }^{-1}$ when classifying moderate-to-vigorous intensity physical activity.

\section{References}

Adolph, A. L., Puyau, M. R., Vohra, F. A., Nicklas, T. A., Zakeri, I. F., \& Butte, N. F. (2012). Validation of uniaxial and triaxial accelerometers for the assessment of physical activity in preschool children. Journal of physical activity \& health, 9, 944-953.

Alhassan, S., Lyden, K., Howe, C., Nwaokelemeh, O., \& Freedson, P. (2012). Accuracy of accelerometer regression models in predicting energy expenditure and METs in children and youth. Pediatric Exercise Science, 24, 519-536.

Bassett, D. R. J., Rowlands, A., \& Trost, S. G. (2012). Calibration and Validation of Wearable Monitors. Medicine and Science in Sports and Exercise, 44, 32-38.

Beets, M. W., Bornstein, D., Dowda, M., \& Pate, R. R. (2011). Compliance With National Guidelines for Physical Activity in U.S. Preschoolers: Measurement and Interpretation. Pediatrics, 127, 658-664.

Cain, K. L., Sallis, J.F., Conway, T.L. Van Dyck, D., \& Calhoon, L. (2013). Using accelerometers in youth physical activity studies: a review of methods. Journal of physical activity \& health $\mathbf{1 0}(3)$.

Cardon, G. M., \& De Bourdeaudhuij, I. M. M. (2008). Are preschool children active enough? Objectively measured physical activity levels. Research Quarterly for Exercise and Sport, 79, 326-332.

Carter, P. J., Taylor, B. J., Williams, S. M., \& Taylor, R. W. (2011). Longitudinal analysis of sleep in relation to BMI and body fat in children: the FLAME study. BMJ: British Medical Journal, 342. doi: http://dx.doi.org/10.1136/bmj.d2712 
Cliff, D. P., \& Okely, A. D. (2007). Comparison of two sets of accelerometer cut-off points for calculating moderate-to-vigorous physical activity in young children. Journal of Physical Activity and Health, 4, 509-513.

Cole, T. J., Bellizzi, M. C., Flegal, K. M., \& Dietz, W. H. (2000). Establishing a standard definition for child overweight and obesity worldwide: international survey. British Medical Journal (Clinical research ed.), 320, 1240-1243.

Colley, R. C., Garriguet, D., Janssen, I., Craig, C. L., Clarke, J., \& Tremblay, M. S. (2011). Physical activity of Canadian children and youth: accelerometer results from the 2007 to 2009 Canadian Health Measures Survey. Health Reports, 22, 15-23.

De Bock, F., Menze, J., Becker, S., Litaker, D., Fischer, J., \& Seidel, I. (2010). Combining Accelerometry and HR for Assessing Preschoolers' Physical Activity. Medicine and Science in Sports and Exercise, 42, 2237-2243.

Department of Health, Physical Activity, Health Improvement and Protection. (2011). Start Active, Stay Active: A Report on Physical Activity from the Four Home Countries' Chief Medical Officers. UK: Department of Health London.

Department of Health and Ageing. (2009). Get Up and Grow: Healthy Eating and Physical Activity for Early Childhood. Physcial activity guidelines for 0-5 year olds. Canberra: Commonwealth of Australia.

Dolinsky, D. H., Brouwer, R. J. N., Østbye, T., Evenson, K. R., \& Siega-Riz, A. M. (2011). Correlates of Sedentary Time and Physical Activity Among PreschoolAged Children. Preventing Chronic Disease, 8, A131.

DuRant, R. H., Baranowski, T., Puhl, J., \& Rhodes, T. (1993). Evaluation of the Children's Activity Rating Scale (CARS) in young children. Medicine and Science in Sports and Exercise, 25, 1415-1421. 
Evenson, K. R., Catellier, D. J., Gill, K., Ondrak, K. S., \& McMurray, R. G. (2008).

Calibration of two objective measures of physical activity for children. Journal of Sports Sciences, 26, 1557 - 1565.

Freedson, P., Bowles, H. R., Troiano, R., \& Haskell, W. (2012). Assessment of Physical Activity Using Wearable Monitors: Recommendations for Monitor Calibration and Use in the Field. Medicine and Science in Sports and Exercise, 44, 1-4.

Heil, D. P. (2006). Predicting Activity Energy Expenditure Using the Actical ${ }^{\circledR}$ Activity Monitor. Research Quarterly for Exercise and Sport, 77, 64-80. doi: $10.1080 / 02701367.2006 .10599333$

Hinkley, T., Salmon, J., Okely, A. D., Crawford, D., Hesketh, K. (2012). Preschoolers Physical Activity, Screen Time, and Compliance with Recommendations. Medicine and Science in Sports and Exercise, 44, 458-465.

Jackson, D. M., Pace, L., \& Speakman, J. R. (2007). The Measurement of Resting Metabolic Rate in Preschool Children. Obesity, 15(8), 1930-1932.

Janssen, X., Cliff, D. P., Okely, A. D., Jones, R. A., Batterham, M., Ekelund, U., Brage, S., Reilly, J. J. (2013). Practical utility and reliability of whole-room calorimetery in young children. British Journal of Nutrition, 109, 1917-1922.

Janssen, X., D. P. Cliff, Reilly, J.J., Hinkley, T., Jones, R.A., Batterham, M., Ekelund, U., Brage, S., Okely, A.D. (2013). Predictive validity and classification accuracy of ActiGraph energy expenditure equations and cut-points in young children" PLoS ONE 8, e79124.

Landis, J. R., \& Koch, G. G. (1977). The measurement of observer agreement for categorical data. Biometrics, 33, 159-174.

Martin, A., McNeil, M., Penpraze, V., Dall, P., Granat, M., Paton, J., \& Reilly, J. (2011). Objective measurement of habitual sedentary behavior in pre-school children: 
comparison of activPAL with actigraph monitors. Pediatric Exercise Science, 23, 468-476.

McClain, J. J., Abraham, T. L., Brusseau Jr, T. A., \& Tudor-Locke, C. (2008). Epoch length and accelerometer outputs in children: comparison to direct observation. Medicine and Science in Sports and Exercise, 40, 2080-2087.

Metz, C. E. (1978). Basic principles of ROC analysis. Seminars in Nuclear Medicine, 8, 283-298.

Oliver, M., Schofield, G. M., \& Kolt, G. S. (2007). Physical Activity in Preschoolers: Understanding Prevalence and Measurement Issues. Sports Medicine, 37, 10451070.

Pate, R. R., Almeida, M. J., McIver, K. L., Pfeiffer, K. A., \& Dowda, M. (2006). Validation and calibration of an accelerometer in preschool children. Obesity, 14, 2000-2006.

Pfeiffer, K. A., McIver, K. L., Dowda, M., Almeida, M., \& Pate, R. R. (2006). Validation and calibration of the Actical accelerometer in preschool children. Medicine and Science in Sports and Exercise, 38, 152-157.

Puhl, J. (1990). Children's Physical Activity Rating Scale (CARS): description and calibration. Research Quarterly for Exercise and Sport, 61, 26-36.

Puyau, M. R., Adolph, A. L., Vohra, F. A., Zakeri, I., \& Butte, N. F. (2004). Prediction of activity energy expenditure using accelerometers in children. [Article]. Medicine and Science in Sports and Exercise, 36, 1625-1631.

Reilly, J. J., Kelly, L. A., Montgomery, C., Jackson, D. M., Slater, C., Grant, S., \& Paton, J. Y. (2006). Validation of Actigraph accelerometer estimates of total energy expenditure in young children. International Journal of Pediatric Obesity, 1, 161167. 
Reilly, J. J., Penpraze, V., Hislop, J., Davies, G., Grant, S., \& Paton, J. Y. (2008).

Objective measurement of physical activity and sedentary behaviour: review with new data. Archives of Disease in Childhood, 93, 614-619.

Schoffelen, P. F. M., Westerterp, K. R., Saris, W. H. M., \& Ten Hoor, F. (1997). A dualrespiration chamber system with automated calibration. Journal of Applied Physiology, 83, 2064-2072.

Schofield, W. N. (1985). Predicting basal metabolic rate, new standards and review of previous work. Human nutrition. Clinical nutrition, 39, 5-41.

Tremblay, M. S., LeBlanc, A. G., Carson, V., Choquette, L., Connor Gorber, S., Dillman, C., . . . Janssen, I. (2012). Canadian physical activity guidelines for the early years (aged 0-4 years). Applied Physiology, Nutrition, and Metabolism, 37, 345-356.

Trost, S. G. (2007). State of the Art Reviews: Measurement of Physical Activity in Children and Adolescents. American Journal of Lifestyle Medicine, 1, 299-314.

Trost, S. G., Loprinzi, P. D., Moore, R., \& Pfeiffer, K. A. (2011). Comparison of accelerometer cut points for predicting activity intensity in youth. Medicine and Science in Sports and Exercise, 43, 1360-1368.

Trost, S. G., Way, R., \& Okely, A. D. (2006). Predictive Validity of Three ActiGraph Energy Expenditure Equations for Children. Medicine and Science in Sports and Exercise, 38, 380-387.

Vale, S., Silva, P., Santos, R., Soares-Miranda, L., \& Mota, J. (2010). Compliance with physical activity guidelines in preschool children. Journal of Sports Sciences, 28, 603-608.

Van Cauwenberghe, E., Jones, R. A., Hinkley, T., Crawford, D., \& Okely, A. D. (2012). Patterns of physical activity and sedentary behaviour in preschool children. 
International Journal of Behavioral Nutrition and Physical Activity, 9. doi: $10.1186 / 1479-5868-9-138$

Van Cauwenberghe, E., Trost, S. G., De Bourdeaudhuij, I., \& Cardon, G. (2011).

Calibration and comparison of accelerometer cut points in preschool children. International Journal of Pediatric Obesity, 6, e582-e589.

Van Cauwenberghe, E., Wooller, L., Mackay, L., Cardon, G., \& Oliver, M. (2012). Comparison of Actical and activPAL measures of sedentary behaviour in preschool children. Journal of Science and Medicine in Sport, 15, 526-531.

Van Puyenbroeck, J., Maes, B., \& Laeremans, P. (2005). Vitessa (Version 0.1): University of Leuven, Belgium.

Weir, J. B. (1949). New methods for calculating metabolic rate with special reference to protein metabolism. The Journal of Physiology, 109, 1-9. 
Table 1. Actical cut-points and equations for children.

\begin{tabular}{|c|c|c|c|c|c|c|}
\hline Author & Sample & Criterion measure & Activities & & \multicolumn{2}{|c|}{$\begin{array}{l}\text { Equation/Cut-point } \\
\text { counts } \cdot 15 \mathrm{~s}^{-} \text {counts } \cdot 60 \mathrm{~s}^{-1} \\
1\end{array}$} \\
\hline Adolph et al. ${ }^{1 *}$ & $\begin{array}{l}\mathrm{n}=64(27 \text { girls, } 37 \text { boys }) \\
\text { Age }=3-5 \text { years } \\
\text { Mean age }=4.5 \text { years }\end{array}$ & Room calorimetry & $\begin{array}{l}\text { Sitting, drawing, playing with toys, } \\
\text { ball play, dance, jogging. }\end{array}$ & $\begin{array}{l}\text { SB } \\
\text { LPA } \\
\text { MVPA }\end{array}$ & $\begin{array}{l}\leq 6 \\
>6 \\
\geq 287\end{array}$ & $\begin{array}{l}\leq 25 \\
>25 \\
\geq 1150\end{array}$ \\
\hline Evenson et al. ${ }^{14}$ & $\begin{array}{l}\mathrm{n}=33(21 \text { girls, } 12 \text { boys }) \\
\text { Age }=5-8 \text { years } \\
\text { Mean age }=7.3 \text { years }\end{array}$ & $\begin{array}{l}\text { Portable metabolic } \\
\text { system }\end{array}$ & $\begin{array}{l}\text { Sit, watch TV, colouring in, slow } \\
\text { walk, stair climbing, dribble } \\
\text { basketball, brisk walk, bicycling, } \\
\text { jumping jacks, running. }\end{array}$ & $\begin{array}{l}\text { SB } \\
\text { LPA } \\
\text { MVPA }\end{array}$ & $\begin{array}{l}\leq 11 \\
>11 \\
\geq 508\end{array}$ & $\begin{array}{l}\leq 44 \\
>44 \\
\geq 2032\end{array}$ \\
\hline Pfeiffer et al. ${ }^{25}$ & $\begin{array}{l}\mathrm{n}=18(11 \text { girls, } 7 \text { boys }) \\
\text { Age }=3-5 \text { years } \\
\text { Mean age }=4.4 \text { years }\end{array}$ & $\begin{array}{l}\text { Portable metabolic } \\
\text { system }\end{array}$ & Sitting, walking, running. & $\begin{array}{l}\mathrm{VO}_{2}=9 \\
\mathrm{SB} \\
\text { LPA } \\
\text { MVPA }\end{array}$ & $\begin{array}{l}73+(0 . \\
\text { NA } \\
<715 \\
\geq 715\end{array}$ & $\begin{array}{l}\text { 7) } x \text { counts } / 15 s \\
\text { NA } \\
<2860 \\
\geq 2860\end{array}$ \\
\hline
\end{tabular}

* developed for 60s epochs; SB, sedentary behaviour; LPA, light intensity physical activity; MVPA, moderate-to-vigorous intensity physical activity 
Table 2. Participant characteristics.

\begin{tabular}{lccc}
\hline & $\begin{array}{c}\text { Total sample } \\
(\mathbf{n}=\mathbf{4 0})\end{array}$ & $\begin{array}{c}\text { Boys } \\
(\mathbf{n = 2 2})\end{array}$ & $\begin{array}{c}\text { Girls } \\
(\mathbf{n = 1 8})\end{array}$ \\
\hline Age (years) & $5.3 \pm 1.0$ & $5.2 \pm 1.0$ & $5.3 \pm 1.1$ \\
Height $(\mathrm{cm})$ & $112.7 \pm 8.1$ & $114.3 \pm 6.2$ & $110.9 \pm 9.7$ \\
Weight $(\mathrm{kg})$ & $20.6 \pm 3.7$ & $21.5 \pm 2.4$ & $19.4 \pm 4.6$ \\
BMI $\left(\mathrm{kg} / \mathrm{m}^{2}\right)$ & $16.1 \pm 1.5$ & $16.5 \pm 1.3$ & $15.5 \pm 1.6$ \\
\% overweight* & 25.0 & 27.2 & 22.2 \\
\hline
\end{tabular}

Values are mean $\pm \mathrm{SD}$; *defined according to Cole et al. (2000). 
Table 3. Median and interquartile values for measured energy expenditure and Actical counts per intensity.

\begin{tabular}{lcccccc}
\hline & \multicolumn{2}{c}{$\begin{array}{c}\text { Energy Expenditure } \\
\text { (kcal/kg/min) }\end{array}$} & \multicolumn{2}{c}{ METs } & \multicolumn{2}{c}{$\begin{array}{c}\text { Actical } \\
\text { (counts/15s) }\end{array}$} \\
& Median & Interquartile & Median & Interquartile & Median & Interquartile \\
\hline SB & 0.0052 & $0.0009-0.0111$ & 1.19 & $1.0-1.3$ & 3 & $1-5$ \\
LPA & 0.0280 & $0.0238-0.0330$ & 1.91 & $1.8-2.1$ & 75 & $32-136$ \\
MVPA & 0.0758 & $0.0624-0.0973$ & 3.5 & $3.1-4.0$ & 787 & $736-1195$ \\
Total & 0.2028 & $0.0153-0.0328$ & 1.6 & $1.5-2.2$ & 53 & $23-311$ \\
\hline \multicolumn{2}{l}{$\begin{array}{l}\text { METs, metabolic equivalents; SB, sedentary behaviour; LPA, light intensity physical activity; MVPA, moderate-to-vigorous intensity physical } \\
\text { activity }\end{array}$}
\end{tabular}


Table 4. Activity energy expenditure for the calorimeter and Pfeiffer Actical equation.

\begin{tabular}{|l|l|c|c|c|c|}
\hline & \multicolumn{2}{|c|}{$\begin{array}{c}\text { Room } \\
\text { calorimetry } \\
\text { (kcal/kg/min) }\end{array}$} & \multicolumn{3}{|c|}{$\begin{array}{c}\text { Pfeiffer } \\
\text { (kcal/kg/min) }\end{array}$} \\
\hline & Mean & sd & Mean & sd & rmse \\
\hline SB & 0.005 & 0.007 & $0.017^{*}$ & 0.004 & 0.015 \\
\hline LPA & 0.030 & 0.008 & $0.023^{*}$ & 0.011 & 0.011 \\
\hline MVPA & 0.081 & 0.024 & 0.080 & 0.027 & 0.028 \\
\hline Total & 0.027 & 0.016 & 0.027 & 0.016 & 0.009 \\
\hline
\end{tabular}

* significant difference with activity energy expenditure measured by room calorimetry $(\mathrm{P}<0.0125)$; SB, sedentary behaviour; LPA, light intensity physical activity; MVPA, moderate-to-vigorous intensity physical activity 
Table 5. Sensitivity (Se\%), Specificity (Sp\%) and area under the ROC curve (ROC-AUC) for the classification of SB, LPA and MVPA using direct observation as criterion measure.

\begin{tabular}{|c|c|c|c|c|c|c|c|c|c|c|c|c|}
\hline & \multicolumn{3}{|c|}{ SB } & \multicolumn{3}{|c|}{ LPA } & \multicolumn{3}{|c|}{ MVPA } & \multicolumn{3}{|c|}{ LMVPA } \\
\hline & $\begin{array}{c}\text { Se\% } \\
(95 \% \mathrm{CI})\end{array}$ & $\begin{array}{c}\text { Sp\% } \\
(95 \% \mathrm{CI})\end{array}$ & $\begin{array}{r}\text { ROC-AUC } \\
(95 \% \mathrm{CI})\end{array}$ & $\begin{array}{c}\text { Se\% } \\
(95 \% \mathrm{CI})\end{array}$ & $\begin{array}{c}\text { Sp\% } \\
(95 \% \mathrm{CI})\end{array}$ & $\begin{array}{r}\text { ROC-AUC } \\
(95 \% \mathrm{CI})\end{array}$ & $\begin{array}{c}\mathrm{Se} \% \\
(95 \% \mathrm{CI})\end{array}$ & $\begin{array}{c}\text { Sp\% } \\
(95 \% \mathrm{CI})\end{array}$ & $\begin{array}{c}\text { ROC-AUC } \\
(95 \% \mathrm{CI})\end{array}$ & $\begin{array}{c}\mathrm{Se} \% \\
(95 \% \mathrm{CI})\end{array}$ & $\begin{array}{c}\text { Sp\% } \\
(95 \% \mathrm{CI})\end{array}$ & $\begin{array}{c}\text { ROC-AUC } \\
(95 \% \mathrm{CI})\end{array}$ \\
\hline \multirow[t]{2}{*}{ Adolph $_{60 \text { s }}$} & 82.5 & 81.1 & 0.82 & 66.2 & 80.2 & 0.73 & 74.3 & 95.3 & 0.85 & 81.1 & 82.5 & 0.82 \\
\hline & $(80.6-84.3)$ & $(79.8-82.2)$ & $(0.81-0.83)$ & $(64.4-68.0)$ & $(78.6-81.6)$ & $(0.72-0.74)$ & $(71.6-76.8)$ & $(94.6-95.9)$ & $(0.83-0.86)$ & $(79.8-82.2)$ & $(80.6-84.3)$ & $(0.81-0.83)$ \\
\hline \multirow[t]{2}{*}{ Adolph $_{15 \mathrm{~s}}$} & 89.1 & 71.4 & 0.80 & 51.9 & 84.3 & 0.68 & 71.0 & 93.6 & 0.82 & 71.4 & 89.1 & 0.80 \\
\hline & $(88.3-89.8)$ & $(70.7-72.1)$ & $(0.80-0.81)$ & $(50.9-52.8)$ & $(83.6-85.0)$ & $(0.68-0.69)$ & $(69.7-72.3)$ & $(93.2-93.9)$ & $(0.82-0.83)$ & $(70.7-72.1)$ & $(88.3-89.8)$ & $(0.80-0.81)$ \\
\hline \multirow[t]{2}{*}{ Evenson $_{15 \mathrm{~s}}$} & 91.0 & 68.0 & 0.79 & 51.0 & 79.1 & 0.65 & 54.9 & 96.0 & 0.75 & 68.0 & 91.0 & 0.79 \\
\hline & $(90.2-91.6)$ & $(67.3-68.7)$ & $(0.79-0.80)$ & $(50.1-51.9)$ & $(78.3-79.8)$ & $(0.64-0.66)$ & $(53.5-56.4)$ & $(95.7-96.3)$ & $(0.75-0.76)$ & $(67.3-68.7)$ & (90.2-91.6) & $(0.79-0.80)$ \\
\hline \multirow[t]{2}{*}{ Pfeiffer $_{15 \mathrm{~s}} *$} & & & & & & & 43.96 & 96.5 & 0.70 & & & \\
\hline & & & & & & & $(42.5-45.4$ & $(96.2-96$. & $(0.70-0.71)$ & & & \\
\hline
\end{tabular}

15s: 15 second epoch; 60s: 60 second epoch; * did not develop a sedentary behaviour or light intensity physical activity cut-point; SB, sedentary behaviour; LPA, light intensity physical activity; MVPA, moderate-to-vigorous intensity physical activity 
Table 6. Sensitivity (Se\%), Specificity (Sp\%) and area under the ROC curve (ROC-AUC) for the classification of SB, LPA and MVPA using EE combined with direct observation as the criterion measure.

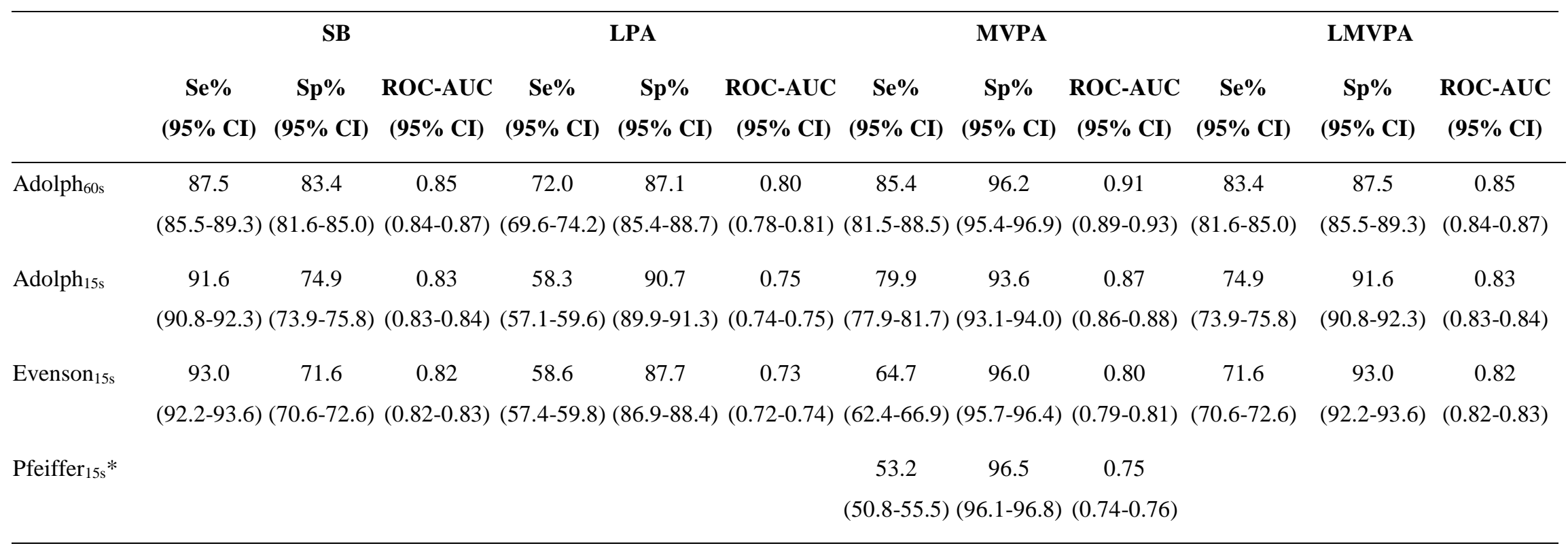

15s: 15 second epoch; ${ }_{60}: 60$ second epoch; * did not develop a sedentary behaviour or light intensity physical activity cut-point; SB, sedentary behaviour; LPA, light intensity physical activity; MVPA, moderate-to-vigorous intensity physical activity 